\title{
Finite-temperature relativistic Landau problem and the relativistic quantum Hall effect
}

\author{
C G Beneventano $\ddagger$ and E M Santangelo $\S$ \\ Departamento de Física, Universidad Nacional de La Plata \\ Instituto de Física de La Plata, UNLP-CONICET \\ C.C. 67, 1900 La Plata, Argentina \\ E-mail: gabriela@obelix.fisica.unlp.edu.ar, \\ mariel@obelix.fisica.unlp.edu.ar
}

\begin{abstract}
This paper presents a study of the free energy and particle density of the relativistic Landau problem, and their relevance to the quantum Hall effect. We study first the zero temperature Casimir energy and fermion number for Dirac fields in a 2+1-dimensional Minkowski space-time,in the presence of a uniform magnetic field perpendicular to the spatial manifold. Then, we go to the finitetemperature problem, with a chemical potential, introduced as a uniform zero component of the gauge potential. By performing a Lorentz boost, we obtain Hall's conductivity in the case of crossed electric and magnetic fields.
\end{abstract}

Subject Classification

PACS: $11.10 . \mathrm{Wx}, 02.30 . \mathrm{Sa}$

\section{Introduction}

The quantization of the Hall conductivity [1] is a remarkable quantum phenomenon, which occurs in two-dimensional electron systems, at low temperatures and strong perpendicular magnetic fields. Most proposed explanations for this phenomenon (for a review see, for instance, 22) rely on one-particle theory and make use of the Kubo formula concerning the conductivity as a linear response function to the external field [3].

It is the aim of this paper to show that, in the context of relativistic field theory, the quantization of the Hall conductivity in multiples of the magnetic flux arises as a consequence of the spin-statistics theorem, which is a straightforward outcome of such a theory.

After this calculation was finished, a very interesting, recently published [4, calculation of the Hall conductivity in relativistic systems was brought to our knowledge. We will compare our results to those obtained in this recent publication in our final comments.

In section 2 we present the theory of Dirac fields in $2+1$ Minkowski space-time, interacting with a magnetic background field perpendicular to the spatial plane, and evaluate the vacuum expectation values of the energy and fermion density.

$\ddagger$ Member of CONICET

$\S$ Member of CONICET 
Section 3 contains the generalities of the same theory in Euclidean 3-dimensional space, and presents the eigenvalues of the corresponding Dirac operator. From such eigenvalues, the partition function is evaluated in section 4

Section 15 contains the resulting free energy and mean particle density at finite temperature, and a study of their zero-temperature limit. In the same section, we perform an adequate Lorentz boost in order to consider the problem of fermions interacting with crossed electric and magnetic fields, and obtain the Hall conductivity.

Some final comments about the case in which the chemical potential $\mu$ coincides with an energy level are presented in section [6] In the same section, we discuss the role that experimental results on the quantum Hall effect can play, as an arena for testing the physical relevance of the phase of the determinant or, equivalently, of the multiplicative anomaly, in view of our discrepancies with reference [4].

A very sketchy presentation of the present study can be found in [5].

\section{Zero-temperature problem}

We will use the metric $(-,+,+)$, natural units $\hbar=c=1$, and choose the following representation for the Dirac matrices: namely,

$$
\gamma_{M}^{0}=i \sigma_{3}, \quad \gamma_{M}^{1}=\sigma_{2} \quad \text { and } \quad \gamma_{M}^{2}=\sigma_{1} .
$$

The Hamiltonian can be determined from the solutions of the Dirac equation $(i \not \partial-e A) \Psi=0$, where $-e$ is the negative charge of the electron. In the Landau gauge $A=(0,0, B x)$, with $B>0$. Thus, after setting $\Psi(t, x, y)=e^{-i E t} \psi(x, y)$, we get the Hamiltonian $H=i \sigma_{1} \partial_{x}-i \sigma_{2} \partial_{y}+\sigma_{2} e B x$. The corresponding eigenvalues can be determined from

$$
\left(\begin{array}{cc}
-E & i \partial_{x}-\partial_{y}-i e B x \\
i \partial_{x}+\partial_{y}+i e B x & -E
\end{array}\right) \psi(x, y)=0 .
$$

In order to solve the above equation, we take

$$
\psi_{k}(x, y)=\left(\begin{array}{c}
\varphi_{k}(x, y) \\
\chi_{k}(x, y)
\end{array}\right)=\frac{1}{\sqrt{2 \pi}}\left(\begin{array}{c}
e^{i k y} \varphi_{k}(x) \\
e^{i k y} \chi_{k}(x)
\end{array}\right) .
$$

This leads to the following system of first order equations

$$
\begin{aligned}
\left(i \partial_{x}-i k-i e B x\right) \chi_{k} & =E \varphi_{k} \\
\left(i \partial_{x}+i k+i e B x\right) \varphi_{k} & =E \chi_{k} .
\end{aligned}
$$

We first solve for the zero mode $E_{0}=0$. In this case, both equations decouple and, after imposing that the eigenfunctions be well-behaved for $x \rightarrow \pm \infty$ we obtain, up to normalization

$$
\psi_{k}(x)=\left(\begin{array}{c}
(e B / \pi)^{1 / 4} \exp \left\{-\frac{1}{2} e B(x+k / e B)^{2}\right\} \\
0
\end{array}\right) .
$$

In the case $E \neq 0$, the normalized eigenfunctions which are well-behaved for all values of $x$ can be written in terms of the Hermite's polynomials $H_{n}(u)[\underline{6}$ as follows

$$
\begin{aligned}
\psi_{k, n}^{ \pm}(x) & =\frac{(e B / \pi)^{1 / 4}}{\sqrt{n ! 2^{n+1}}} \exp \left\{-\frac{1}{2} e B(x+k / e B)^{2}\right\} \\
& \times\left(\begin{array}{l} 
\pm H_{n}(\sqrt{e B}(x+k / e B)) \\
i \sqrt{2 n} H_{n-1}(\sqrt{e B}(x+k / e B))
\end{array}\right)
\end{aligned}
$$


with corresponding eigenvalues

$$
E_{n}= \pm \sqrt{2 n e B}, \quad n=1, \ldots, \infty .
$$

We notice that each eigenvalue exhibits the well known Landau's degeneracy per unit area: namely,

$$
\Delta_{L}=\frac{e B}{2 \pi}
$$

Had we chosen the other non-equivalent representation of the gamma matrices in $2+1$ dimensions, exactly the same spectrum would have been obtained obtained, the only difference being the chirality of eigenfunctions.

The vacuum expectation value of the energy per unit area, defined through a zeta function regularization (see, for example, [7, and references therein), is given by

$$
\left.E_{C}=-\frac{\Delta_{L}}{2} \sum_{E_{n} \neq 0}\left|E_{n}\right|^{-s}\right\rfloor_{s=-1}
$$

In the present case, we have ( $\alpha$ is an arbitrary parameter with mass dimension, introduced to render the complex powers dimensionless)

$\left.E_{C}(B)=-\frac{\Delta_{L} \alpha}{2} 2 \sum_{n=1}^{\infty}\left(\frac{\sqrt{2 n e B}}{\alpha}\right)^{-s}\right\rfloor_{s=-1}=-\Delta_{L} \sqrt{2 e B} \zeta_{R}\left(-\frac{1}{2}\right)$.

Always in the zeta-function regularization framework, the fermion number is 8

$$
\left.N(B)=-\frac{\Delta_{L}}{2}\left(\sum_{E_{n}>0}\left|E_{n}\right|^{-s}-\sum_{E_{n}<0}\left|E_{n}\right|^{-s}\right)\right\rfloor_{s=0}+N_{0},
$$

where $N_{0}$ is the contribution coming from zero modes.

In our case, the nonvanishing spectrum is symmetric. So, only the zero mode, which is charge self-conjugate, contributes. This gives as a result [8]

$$
N(B)= \pm \frac{\Delta_{L}}{2}
$$

Or, equivalently, for the vacuum expectation value of the charge density

$$
j^{0}(B)=\mp e \frac{\Delta_{L}}{2} .
$$

The sign indetermination is a natural consequence of the twofold vacuum degeneracy.

\section{The theory at finite temperature with chemical potential}

In order to study the effect of temperature, we go to Euclidean space, with the metric $(+,+,+)$. To this end, we take the Euclidean gamma matrices to be $\gamma_{0}=i \gamma_{M}^{0}=-\sigma_{3}$, $\gamma_{1}=\gamma_{M}^{1}=\sigma_{2}, \gamma_{2}=\gamma_{M}^{2}=\sigma_{1}$. We will follow [9] in introducing the chemical potential as an imaginary $A_{0}=-i \frac{\mu}{e}$ in Euclidean space. Thus, the partition function in the grand-canonical ensemble is given by

$$
\ln Z=\ln \operatorname{det}(i \not \partial-e A) \text {. }
$$


In order to evaluate the partition function in the zeta regularization approach [10, we first determine the eigenfunctions, and the corresponding eigenvalues, of the Dirac operator, in the same gauge used in the previous section, i.e, we solve

$$
\left[-i \sigma_{3}\left(\partial_{\tau}+\mu\right)+i \sigma_{2} \partial_{x}+\sigma_{1}\left(i \partial_{y}-e B x\right)\right] \Psi=\omega \Psi
$$

or, after writing $\Psi(\tau, x, y)=\left(\begin{array}{c}\Phi(\tau, x, y) \\ \Xi(\tau, x, y)\end{array}\right)$,

$$
\left(\begin{array}{cc}
-i\left(\partial_{\tau}+\mu\right) & \partial_{x}+i \partial_{y}-e B x \\
-\partial_{x}+i \partial_{y}-e B x & i\left(\partial_{\tau}+\mu\right)
\end{array}\right)\left(\begin{array}{c}
\Phi(\tau, x, y) \\
\Xi(\tau, x, y)
\end{array}\right)=\omega\left(\begin{array}{c}
\Phi(\tau, x, y) \\
\Xi(\tau, x, y)
\end{array}\right) .
$$

In order to satisfy antiperiodic boundary conditions in the $\tau$ direction, we propose

$$
\Psi_{k, l}(\tau, x, y)=\frac{e^{i \lambda_{l} \tau} e^{i k y}}{\sqrt{2 \pi \beta}} \psi_{k, l}(x),
$$

with

$$
\lambda_{l}=(2 l+1) \frac{\pi}{\beta},
$$

where $\beta=\frac{1}{T}$ is the inverse temperature.

After doing so, and writing

$$
\psi_{k, l}(x)=\left(\begin{array}{c}
\varphi_{k, l}(x) \\
\chi_{k, l}(x)
\end{array}\right)
$$

we have, for each $k, l$,

$$
\begin{aligned}
& \left(\partial_{x}-k-e B x\right) \chi_{k, l}=\left(\omega-\tilde{\lambda}_{l}\right) \varphi_{k, l} \\
& \left(-\partial_{x}-k-e B x\right) \varphi_{k, l}=\left(\omega+\tilde{\lambda}_{l}\right) \chi_{k, l},
\end{aligned}
$$

where $\tilde{\lambda}_{l}=\lambda_{l}-i \mu$.

There are two types of eigenvalues and corresponding eigenfunctions

1) $\omega_{l}=\tilde{\lambda}_{l}$, with $l=-\infty, \ldots, \infty$, and corresponding normalized eigenfunctions

$$
\psi_{k, l}(x)=\left(\begin{array}{c}
\left(\frac{e B}{\pi}\right)^{\frac{1}{4}} e^{-\frac{e B}{2}\left(x+\frac{k}{e B}\right)^{2}} \\
0
\end{array}\right) .
$$

Note that these eigenvalues are not square roots of the eigenvalues of the squared operator. They will eventually lead to a "spectral asymmetry" || and, thus, to a phase of the determinant, which will be studied in detail in the next section.

2) $\omega_{l, n}= \pm \sqrt{\tilde{\lambda}_{l}^{2}+2 n e B}$, with $n=1, \ldots, \infty, l=-\infty, \ldots, \infty$, and corresponding normalized eigenfunctions

$\psi_{k, l, n}(x)=A_{k, l, n}\left(\begin{array}{l}-\frac{\left(\omega_{l, n}+\tilde{\lambda}_{l}\right)}{2 n \sqrt{e B}} e^{-\frac{e B}{2}\left(x+\frac{k}{e B}\right)^{2}} H_{n}\left(\sqrt{e B}\left(x+\frac{k}{e B}\right)\right) \\ e^{-\frac{e B}{2}\left(x+\frac{k}{e B}\right)^{2}} H_{n-1}\left(\sqrt{e B}\left(x+\frac{k}{e B}\right)\right)\end{array}\right)$,

where

$$
A_{k, l, n}=\left(\frac{e B}{\pi}\right)^{\frac{1}{4}} \frac{2^{\frac{1-n}{2}}}{[(n-1) !]^{\frac{1}{2}}}\left[\frac{2 n e B}{2 n e B+\left|\omega_{l, n}+\tilde{\lambda}_{l}\right|^{2}}\right]^{\frac{1}{2}} .
$$

\| Here, quotation marks are due to the fact that, the Dirac operator not being self-adjoint, we have a complex spectrum. 
In all cases, the degeneracy per unit area is again given by $\Delta_{L}$ in equation (8). Choosing the other nonequivalent representation of the gamma matrices leads to a change in the eigenvalues of type 1 ), which are replaced by $\omega_{l}=-\tilde{\lambda}_{l}$. However, as will be discussed below, this doesn't lead to a change in our physical predictions.

\section{Evaluation of the partition function at finite temperature and chemical potential}

The partition function, in the zeta regularization scheme [10], is given by

$$
\left.\log \mathcal{Z}=-\frac{d}{d s}\right\rfloor_{s=0} \zeta\left(s, \frac{i \not \partial-e A}{\alpha}\right) .
$$

As in the previous section, $\alpha$ is a parameter with mass dimension, introduced to render the $\zeta$-function dimensionless.

We must consider two contributions to $\log \mathcal{Z}$, respectively coming from eigenvalues of type 1) and 2) in the previous section, i.e.,

$$
\left.\Delta_{1}(\mu)=-\frac{d}{d s}\right\rfloor_{s=0} \zeta_{1}(s, \mu),
$$

where

$$
\zeta_{1}(s, \mu)=\Delta_{L} \sum_{l=-\infty}^{\infty}\left[(2 l+1) \frac{\pi}{\alpha \beta}-i \frac{\mu}{\alpha}\right]^{-s}
$$

and

$$
\Delta_{2}(\mu, B)=-\left.\frac{d}{d s}\right|_{s=0} \zeta_{2}(s, \mu, B),
$$

where

$$
\zeta_{2}(s, \mu, B)=\left(1+(-1)^{-s}\right) \Delta_{L} \sum_{\substack{n=1 \\ l=-\infty}}^{\infty}\left[\frac{2 n e B}{\alpha^{2}}+\left((2 l+1) \frac{\pi}{\alpha \beta}-i \frac{\mu}{\alpha}\right)^{2}\right]^{-\frac{s}{2}} .
$$

The contribution $\Delta_{1}(\mu)$ can be evaluated at once for the whole $\mu$-range. The analytic extension of $\zeta_{1}(s, \mu)$ can be achieved as follows (for a similar calculation, see 11])

$$
\begin{aligned}
\zeta_{1}(s, \mu) & =\Delta_{L} \sum_{l=-\infty}^{\infty}\left[(2 l+1) \frac{\pi}{\alpha \beta}-i \frac{\mu}{\alpha}\right]^{-s} \\
& =\Delta_{L}\left(\frac{2 \pi}{\alpha \beta}\right)^{-s}\left[\sum_{l=0}^{\infty}\left[\left(l+\frac{1}{2}\right)-i \frac{\mu \beta}{2 \pi}\right]^{-s}+\sum_{l=0}^{\infty}\left[-\left(l+\frac{1}{2}\right)-i \frac{\mu \beta}{2 \pi}\right]^{-s}\right] \\
& =\Delta_{L}\left(\frac{2 \pi}{\alpha \beta}\right)^{-s}\left[\zeta_{H}\left(s, \frac{1}{2}-\frac{i \mu \beta}{2 \pi}\right)+\sum_{l=0}^{\infty}\left[-\left(l+\frac{1}{2}\right)-i \frac{\mu \beta}{2 \pi}\right]^{-s}\right] .
\end{aligned}
$$

Now, in order to write the second term as a Hurwitz zeta, we must relate the eigenvalues with negative real part to those with positive one without, in so doing, 
going through zeros in the argument of the power. Otherwise stated, we must select a cut in the complex $\omega$ plane 12 . This requirement determines a definite value of $(-1)^{-s}$, i.e., $(-1)^{-s}=e^{i \pi \operatorname{sign}(\mu) s}$. Taking this into account, we finally have

$\zeta_{1}(s, \mu)=\Delta_{L}\left(\frac{2 \pi}{\beta \alpha}\right)^{-s}\left[\zeta_{H}\left(s, \frac{1}{2}-\frac{i \mu \beta}{2 \pi}\right)+e^{i \pi \operatorname{sign}(\mu) s} \zeta_{H}\left(s, \frac{1}{2}+\frac{i \mu \beta}{2 \pi}\right)\right]$.

From this last expression, the contribution $\Delta_{1}(\mu)$ to $\log \mathcal{Z}$ can be obtained. It is given by

$$
\begin{aligned}
\Delta_{1}(\mu) & =-\Delta_{L}\left[\zeta_{H}^{\prime}\left(0, \frac{1}{2}-\frac{i \mu \beta}{2 \pi}\right)+\zeta_{H}^{\prime}\left(0, \frac{1}{2}+\frac{i \mu \beta}{2 \pi}\right)+i \pi \operatorname{sign}(\mu) \zeta_{H}\left(0, \frac{1}{2}+\frac{i \mu \beta}{2 \pi}\right)\right] \\
& =\Delta_{L}\left\{\log \left(2 \cosh \left(\frac{\mu \beta}{2}\right)\right)-\frac{|\mu| \beta}{2}\right\} .
\end{aligned}
$$

As commented in advance, the other nonequivalent representation of the gamma matrices leads to the same result for this contribution. In fact, even though this part of the spectrum changes sign, such change is compensated by the selection of the cut in the $\omega$-plane. In this case, one has

$\zeta_{1}(s, \mu)=\Delta_{L}\left(\frac{2 \pi}{\beta \alpha}\right)^{-s}\left[\zeta_{H}\left(s, \frac{1}{2}+\frac{i \mu \beta}{2 \pi}\right)+e^{-i \pi \operatorname{sign}(\mu) s} \zeta_{H}\left(s, \frac{1}{2}-\frac{i \mu \beta}{2 \pi}\right)\right]$,

which also leads to (28).

The analytic extension of $\zeta_{2}(s, \mu, B)$ requires a separate consideration of different $\mu$ ranges. We study in detail three of these cases. The generalization to arbitrary $\mu$-ranges will be evident from these results.

4.1. $\mu^{2}<2 e B$

$\zeta_{2}(s, \mu, B)=\left(1+(-1)^{-s}\right) \Delta_{L} \sum_{\substack{n=1 \\ l=-\infty}}^{\infty}\left[\frac{2 n e B}{\alpha^{2}}+\left((2 l+1) \frac{\pi}{\alpha \beta}-i \frac{\mu}{\alpha}\right)^{2}\right]^{-\frac{s}{2}}$.

Making use of the Mellin transform, this can be written as

$$
\zeta_{2}(s, \mu, B)=\frac{\left(1+(-1)^{-s}\right) \Delta_{L}}{\Gamma\left(\frac{s}{2}\right)} \int_{0}^{\infty} d t t^{\frac{s}{2}-1} \sum_{\substack{n=1 \\ l=-\infty}}^{\infty} e^{-t\left[\frac{2 n e B}{\alpha^{2}}+\left((2 l+1) \frac{\pi}{\alpha \beta}-i \frac{\mu}{\alpha}\right)^{2}\right]}
$$

or, equivalently,

$$
\begin{aligned}
\zeta_{2}(s, \mu, B) & =\frac{\left(1+(-1)^{-s}\right) \Delta_{L}}{\Gamma\left(\frac{s}{2}\right)} \sum_{n=1}^{\infty} \int_{0}^{\infty} d t t^{\frac{s}{2}-1} e^{-t\left[\frac{2 n e B}{\alpha^{2}}+\left(\frac{\pi}{\alpha \beta}-\frac{i \mu}{\alpha}\right)^{2}\right]} \\
& \times \Theta_{3}\left(\frac{-2 t}{\alpha \beta}\left(\frac{\pi}{\alpha \beta}-\frac{i \mu}{\alpha}\right), \frac{4 \pi t}{(\alpha \beta)^{2}}\right)
\end{aligned}
$$

where we have used the definition of the Jacobi theta function

$$
\Theta_{3}(z, x)=\sum_{l=-\infty}^{\infty} e^{-\pi x l^{2}} e^{2 \pi z l}
$$


To proceed, we will use the inversion formula for the Jacobi function

$$
\Theta_{3}(z, x)=\frac{1}{\sqrt{x}} e^{\left(\frac{\pi z^{2}}{x}\right)} \Theta_{3}\left(\frac{z}{i x}, \frac{1}{x}\right),
$$

thus getting

$$
\begin{aligned}
\zeta_{2}(s, \mu, B) & =\frac{\left(1+(-1)^{-s}\right) \Delta_{L} \alpha \beta}{2 \sqrt{\pi} \Gamma\left(\frac{s}{2}\right)} \sum_{n=1}^{\infty} \int_{0}^{\infty} d t t^{\frac{s-1}{2}-1} e^{-t \frac{2 n e B}{\alpha^{2}}} \\
& \times \Theta_{3}\left(\frac{i}{2}+\frac{\mu \beta}{2 \pi}, \frac{(\alpha \beta)^{2}}{4 \pi t}\right) .
\end{aligned}
$$

Applying once more the definition (32), we have

$$
\begin{aligned}
\zeta_{2}(s, \mu, B) & =\frac{\left(1+(-1)^{-s}\right) \Delta_{L} \alpha \beta}{2 \sqrt{\pi} \Gamma\left(\frac{s}{2}\right)}\left\{\int_{0}^{\infty} d t t^{\frac{s-1}{2}-1} \sum_{n=1}^{\infty} e^{-t \frac{2 n e B}{\alpha^{2}}}\right. \\
& \left.+2 \int_{0}^{\infty} d t t^{\frac{s-1}{2}-1} \sum_{n, l=1}^{\infty}(-1)^{l} \cosh (\mu \beta l) e^{-t \frac{2 n e B}{\alpha^{2}}-\frac{(\alpha \beta l)^{2}}{4 t}}\right\} .
\end{aligned}
$$

After performing the integrals [6], we obtain

$$
\begin{aligned}
\zeta_{2}(s, \mu, B) & =\frac{\left(1+(-1)^{-s}\right) \Delta_{L} \alpha \beta}{2 \sqrt{\pi} \Gamma\left(\frac{s}{2}\right)}\left[\Gamma\left(\frac{s-1}{2}\right)\left(\frac{2 e B}{\alpha^{2}}\right)^{\frac{1-s}{2}} \zeta_{R}\left(\frac{s-1}{2}\right)\right. \\
& \left.+4 \sum_{n, l=1}^{\infty}(-1)^{l}\left(\frac{l^{2} \alpha^{4} \beta^{2}}{8 n e B}\right)^{\frac{s-1}{4}} \cosh (\mu \beta l) K_{\frac{s-1}{2}}\left(\sqrt{2 n e B \beta^{2} l^{2}}\right)\right]
\end{aligned}
$$

or, making the simple pole of the $\Gamma$ function explicit

$$
\begin{aligned}
\zeta_{2}(s, \mu, B) & =\frac{\left(1+(-1)^{-s}\right) \Delta_{L} \alpha \beta s}{4 \sqrt{\pi} \Gamma\left(\frac{s+2}{2}\right)}\left[\Gamma\left(\frac{s-1}{2}\right)\left(\frac{2 e B}{\alpha^{2}}\right)^{\frac{1-s}{2}} \zeta_{R}\left(\frac{s-1}{2}\right)\right. \\
& \left.+4 \sum_{n, l=1}^{\infty}(-1)^{l}\left(\frac{l^{2} \alpha^{4} \beta^{2}}{8 n e B}\right)^{\frac{s-1}{4}} \cosh (\mu \beta l) K_{\frac{s-1}{2}}\left(\sqrt{2 n e B \beta^{2} l^{2}}\right)\right] .
\end{aligned}
$$

From this expression, the contribution $\Delta_{2}$ to the partition function can be readily obtained, since the factor accompanying $s$ is finite at $s=0$

$$
\begin{aligned}
\Delta_{2}(\mu, B) & =\frac{-\Delta_{L} \beta}{2 \sqrt{\pi}}\left[\Gamma\left(-\frac{1}{2}\right) \sqrt{2 e B} \zeta_{R}\left(-\frac{1}{2}\right)\right. \\
& \left.+4 \sum_{n, l=1}^{\infty}(-1)^{l}\left(\frac{l^{2} \beta^{2}}{8 n e B}\right)^{-\frac{1}{4}} \cosh (\mu \beta l) K_{-\frac{1}{2}}\left(\sqrt{2 n e B \beta^{2} l^{2}}\right)\right] .
\end{aligned}
$$

After using that

$$
K_{-\frac{1}{2}}(x)=\sqrt{\frac{\pi}{2 x}} e^{-x},
$$

it can be written as

$$
\begin{aligned}
\Delta_{2}(\mu, B) & =\Delta_{L} \beta\left[\sqrt{2 e B} \zeta_{R}\left(-\frac{1}{2}\right)\right. \\
& \left.-\frac{2}{\beta} \sum_{n, l=1}^{\infty} \frac{(-1)^{l}}{l} \cosh (\mu \beta l) e^{-\sqrt{2 n e B} \beta l}\right] .
\end{aligned}
$$


The sum over $l$ can be explicitly performed, to obtain

$$
\begin{aligned}
\Delta_{2}(\mu, B) & =\Delta_{L} \beta\left[\sqrt{2 e B} \zeta_{R}\left(-\frac{1}{2}\right)\right. \\
& \left.+\frac{1}{\beta} \sum_{n=1}^{\infty} \log \left(1+e^{-2 \sqrt{2 n e B} \beta}+2 \cosh (\mu \beta) e^{-\sqrt{2 n e B} \beta}\right)\right] .
\end{aligned}
$$

Finally, adding the contributions given by equations (28) and (40) we obtain, for the partition function in the range $\mu^{2} \leq 2 e B$

$$
\begin{aligned}
\log Z & =\Delta_{L}\left\{\log \left(2 \cosh \left(\frac{\mu \beta}{2}\right)\right)-\frac{|\mu| \beta}{2}+\beta \sqrt{2 e B} \zeta_{R}\left(-\frac{1}{2}\right)\right. \\
& \left.+\sum_{n=1}^{\infty} \log \left(1+e^{-2 \sqrt{2 n e B} \beta}+2 \cosh (\mu \beta) e^{-\sqrt{2 n e B} \beta}\right)\right\} .
\end{aligned}
$$

4.2. $2 e B<\mu^{2}<4 e B$

As before, we have

$$
\zeta_{2}(s, \mu, B)=\left(1+(-1)^{-s}\right) \Delta_{L} \sum_{\substack{n=1 \\ l=-\infty}}^{\infty}\left[\frac{2 n e B}{\alpha^{2}}+\left((2 l+1) \frac{\pi}{\alpha \beta}-i \frac{\mu}{\alpha}\right)^{2}\right]^{-\frac{s}{2}} .
$$

However, in this range of $\mu$, the contribution due to $n=1$ is given by

$$
\Delta_{2}^{n=1}(\mu, B)=-\left.\frac{d}{d s}\right|_{s=0} \zeta_{2}^{n=1}(s, \mu, B),
$$

where

$$
\zeta_{2}^{n=1}(s, \mu, B)=\left(1+(-1)^{-s}\right) \Delta_{L} \sum_{l=-\infty}^{\infty}\left[\frac{2 e B}{\alpha^{2}}+\left((2 l+1) \frac{\pi}{\alpha \beta}-i \frac{\mu}{\alpha}\right)^{2}\right]^{-\frac{s}{2}}
$$

The analytic extension of this expression must be performed in a different way. In fact, the expression cannot be written in terms of a unique Mellin transform, since its real part is not always positive (note, in connection with this that, for $n=1$, equation (37) diverges). Instead, it can be evaluated as follows

$$
\begin{aligned}
\zeta_{2}^{n=1}(s, \mu, B) & =\frac{\left(1+(-1)^{-s}\right)}{\alpha^{-s}} \Delta_{L} \sum_{l=0}^{\infty}\left[2 e B+\left((2 l+1) \frac{\pi}{\beta}-i \mu\right)^{2}\right]^{-\frac{s}{2}} \\
& +\mu \rightarrow-\mu \\
& =\frac{\left(1+(-1)^{-s}\right)}{\alpha^{-s}} \Delta_{L} \sum_{l=0}^{\infty}\left\{\left[i \sqrt{2 e B}+(2 l+1) \frac{\pi}{\beta}-i \mu\right]^{-\frac{s}{2}}\right. \\
& \left.\times\left[-i \sqrt{2 e B}+(2 l+1) \frac{\pi}{\beta}-i \mu\right]^{-\frac{s}{2}}\right\}+\mu \rightarrow-\mu .
\end{aligned}
$$

This can be written as a product of two Mellin transforms 


$$
\begin{aligned}
\zeta_{2}^{n=1}(s, \mu, B) & =\frac{\left(1+(-1)^{-s}\right)}{\alpha^{-s}\left[\Gamma\left(\frac{s}{2}\right)\right]^{2}} \Delta_{L} \sum_{l=0}^{\infty} \int_{0}^{\infty} d t t^{\frac{s}{2}-1} e^{-\left[(2 l+1) \frac{\pi}{\beta}-i \mu+i \sqrt{2 e B}\right]} t \\
& \times \int_{0}^{\infty} d z z^{\frac{s}{2}-1} e^{-\left[(2 l+1) \frac{\pi}{\beta}-i \mu-i \sqrt{2 e B}\right] z}+\mu \rightarrow-\mu
\end{aligned}
$$

or, after a change of variables

$$
\begin{aligned}
\zeta_{2}^{n=1}(s, \mu, B) & =\frac{\left(1+(-1)^{-s}\right)}{2 \alpha^{-s}\left[\Gamma\left(\frac{s}{2}\right)\right]^{2}} \Delta_{L} \sum_{l=0}^{\infty} \int_{0}^{\infty} d z \int_{-z}^{z} d t\left[\frac{z^{2}-t^{2}}{4}\right]^{\frac{s}{2}-1} \\
& \times e^{-\left[(2 l+1) \frac{\pi}{\beta}-i \mu\right] z} e^{-i \sqrt{2 e B} t}+\mu \rightarrow-\mu
\end{aligned}
$$

Now, calling $x=\frac{t}{z}$, one has

$$
\begin{aligned}
\zeta_{2}^{n=1}(s, \mu, B) & =\frac{\left(1+(-1)^{-s}\right)}{2 \alpha^{-s}\left[\Gamma\left(\frac{s}{2}\right)\right]^{2}} \Delta_{L} \sum_{l=0}^{\infty} \int_{0}^{\infty} d z z\left[\frac{z^{2}}{4}\right]^{\frac{s}{2}-1} e^{-\left[(2 l+1) \frac{\pi}{\beta}-i \mu\right] z} \\
& \times \int_{-1}^{1} d x\left[1-x^{2}\right]^{\frac{s}{2}-1} e^{-i \sqrt{2 e B} z x}+\mu \rightarrow-\mu .
\end{aligned}
$$
obtain

Finally, the $x$-integral and the sum of the geometric series can be performed to

$$
\begin{aligned}
\zeta_{2}^{n=1}(s, \mu, B) & =\frac{\left(1+(-1)^{-s}\right) \sqrt{\pi}}{2 \alpha^{-s} \Gamma\left(\frac{s}{2}\right)} \Delta_{L}(2 \sqrt{2 e B})^{\frac{1-s}{2}} \\
& \times \int_{0}^{\infty} d z z^{\frac{s-1}{2}} J_{\frac{s-1}{2}}(\sqrt{2 e B} z) \frac{e^{i \mu z}}{\sinh \left(\frac{\pi z}{\beta}\right)}+\mu \rightarrow-\mu .
\end{aligned}
$$

Now, the integral in this expression diverges at $z=0$. In order to isolate such divergence, we add and subtract the first term in the series expansion of the Bessel function, thus getting the following two pieces

$$
\begin{aligned}
\zeta_{2,(1)}^{n=1}(s, \mu, B) & =\frac{\left(1+(-1)^{-s}\right) \sqrt{\pi} s}{4 \alpha^{-s} \Gamma\left(\frac{s}{2}+1\right)} \Delta_{L}(2 \sqrt{2 e B})^{\frac{1-s}{2}} \\
& \times \int_{0}^{\infty} d z z^{\frac{s-1}{2}}\left[J_{\frac{s-1}{2}}(\sqrt{2 e B} z)-\frac{\left(\frac{\sqrt{2 e B} z}{2}\right)^{\frac{s-1}{2}}}{\Gamma\left(\frac{s+1}{2}\right)}\right] \frac{e^{i \mu z}}{\sinh \left(\frac{\pi z}{\beta}\right)} \\
& +\mu \rightarrow-\mu,
\end{aligned}
$$

and

$\zeta_{2,(2)}^{n=1}(s, \mu, B)=\frac{\left(1+(-1)^{-s}\right) \sqrt{\pi}}{2^{s} \alpha^{-s} \Gamma\left(\frac{s}{2}\right) \Gamma\left(\frac{s+1}{2}\right)} \Delta_{L} \int_{0}^{\infty} d z z^{s-1} \frac{e^{i \mu z}}{\sinh \left(\frac{\pi z}{\beta}\right)}+\mu \rightarrow-\mu$.

The contribution of equation (50) to the partition function, defined as in equation (43) can be easily evaluated by noticing that the factor multiplying $s$ is finite at $s=0$. Thus, one has

$\Delta_{2,(1)}^{n=1}(\mu, B)=-\Delta_{L} \int_{0}^{\infty} d z z^{-1}[\cos (\sqrt{2 e B} z)-1] \frac{e^{i \mu z}}{\sinh \left(\frac{\pi z}{\beta}\right)}+\mu \rightarrow-\mu$, 
where we have used that $J_{-\frac{1}{2}}(\sqrt{2 e B} z)=\sqrt{\frac{2}{\pi \sqrt{2 e B} z}} \cos (\sqrt{2 e B} z)$. Now, in the term with $\mu \rightarrow-\mu$, one can change $z \rightarrow-z$ to obtain

$$
\Delta_{2,(1)}^{n=1}(\mu, B)=-\Delta_{L} \int_{-\infty}^{\infty} d z z^{-1}[\cos (\sqrt{2 e B} z)-1] \frac{e^{i \mu z}}{\sinh \left(\frac{\pi z}{\beta}\right)} .
$$

This last integral is easy to evaluate in the complex plane, by carefully taking into account the sign of $\mu$, as well as well as the fact that $2 e B<\mu^{2}$ in closing the integration path, to obtain

$\Delta_{2,(1)}^{n=1}(\mu, B)=-2 \Delta_{L} \sum_{l=1}^{\infty}\left[\frac{(-1)^{l}}{l} \cosh (\sqrt{2 e B} \beta l) e^{-|\mu| \beta l}+\frac{(-1)^{l+1}}{l} e^{-|\mu| \beta l}\right]$

or, after summing the series

$$
\begin{aligned}
\Delta_{2,(1)}^{n=1}(\mu, B) & =\Delta_{L}\left\{\log \left(1+e^{-2|\mu| \beta}+2 \cosh (\sqrt{2 e B} \beta) e^{-|\mu| \beta}\right)\right. \\
& \left.+|\mu| \beta-2 \log \left(2 \cosh \left(\frac{\mu \beta}{2}\right)\right)\right\} .
\end{aligned}
$$

In order to obtain the contribution coming from (51), the integral can be evaluated for $\Re s>1$, which gives

$$
\begin{aligned}
\zeta_{2,(2)}^{n=1}(s, \mu, B) & =\frac{\left(1+(-1)^{-s}\right) \Gamma(s) \sqrt{\pi}(\alpha \beta)^{s} \Delta_{L}}{(2 \pi)^{s} 2^{s-1} \Gamma\left(\frac{s}{2}\right) \Gamma\left(\frac{s+1}{2}\right)} \\
& \times\left[\zeta_{H}\left(s, \frac{1}{2}\left(1-\frac{i \mu \beta}{\pi}\right)\right)+\zeta_{H}\left(s, \frac{1}{2}\left(1+\frac{i \mu \beta}{\pi}\right)\right)\right],
\end{aligned}
$$

where $\zeta_{H}(s, x)$ is the Hurwitz zeta function. The contribution to the partition function can now be evaluated by using that $\zeta_{H}\left(0, \frac{1}{2}\left(1-\frac{i \mu \beta}{\pi}\right)\right)+\zeta_{H}\left(0, \frac{1}{2}\left(1+\frac{i \mu \beta}{\pi}\right)=0\right.$ and the well known value of $\left.-\frac{d}{d s}\right\rfloor_{s=0} \zeta_{H}(s, x)[\underline{6}$, to obtain

$$
\Delta_{2,(2)}^{n=1}(\mu, B)=2 \Delta_{L} \log \left(2 \cosh \left(\frac{\mu \beta}{2}\right)\right) \text {. }
$$

Summing up the contributions in equations (28), (55) and (57), as well as the contribution coming from $n \geq 2$, evaluated as in the previous subsection, one gets for the partition function

$$
\begin{aligned}
\log Z & =\Delta_{L}\left\{\log \left(2 \cosh \left(\frac{\mu \beta}{2}\right)\right)+\frac{|\mu| \beta}{2}\right. \\
& +\log \left(1+e^{-2|\mu| \beta}+2 \cosh (\sqrt{2 e B} \beta) e^{-|\mu| \beta}\right)+\beta \sqrt{2 e B}\left(\zeta_{R}\left(-\frac{1}{2}\right)-1\right) \\
& \left.+\sum_{n=2}^{\infty} \log \left(1+e^{-2 \sqrt{2 n e B} \beta}+2 \cosh (\mu \beta) e^{-\sqrt{2 n e B} \beta}\right)\right\}
\end{aligned}
$$

At first sight, this result looks different from the one corresponding to $\mu^{2}<2 e B$ (equation (41)). However, it is easy to see that both expressions coincide. The only difference is that (58) explicitly isolates the zero-temperature behavior from finitetemperature corrections for this range of $\mu$. 
4.3. $\mu^{2}=2 e B$

In this case, the analytical extension can be performed, exactly as in the previous subsection, up to equation (52). Then, the term with $\mu \rightarrow-\mu$ can be explicitly summed, which gives

$\Delta_{2,(1)}^{n=1}(|\mu|=\sqrt{2 e B}, B)=-\Delta_{L} \int_{0}^{\infty} d z z^{-1}[\cos (\sqrt{2 e B} z)-1] \frac{2 \cos (\sqrt{2 e B} z)}{\sinh \left(\frac{\pi z}{\beta}\right)}$.

This integral can be found in [], and it gives as a result

$\Delta_{2,(1)}^{n=1}(|\mu|=\sqrt{2 e B}, B)=\Delta_{L}\left[\log (\cosh (\sqrt{2 e B} \beta))-2 \log \left(\cosh \left(\frac{\sqrt{2 e B} \beta}{2}\right)\right)\right]$.

The contribution $\Delta_{2,(2)}^{n=1}(|\mu|=\sqrt{2 e B}, B)$ can be evaluated exactly as in the previous subsection. It is given by

$$
\Delta_{2,(2)}^{n=1}(|\mu|=\sqrt{2 e B}, B)=2 \Delta_{L} \log \left(2 \cosh \left(\frac{\sqrt{2 e B} \beta}{2}\right)\right) .
$$

These two contributions, together with those coming from $n \geq 2$ and from equation (28), finally give for the partition function at this particular value of $\mu$ the same result as (41) or (58) evaluated at $\mu^{2}=2 e B$.

Thus, for any range of $\mu$, one has

$$
\begin{aligned}
\log Z & =\Delta_{L}\left\{\log \left(2 \cosh \left(\frac{\mu \beta}{2}\right)\right)-\frac{|\mu| \beta}{2}+\beta \sqrt{2 e B} \zeta_{R}\left(-\frac{1}{2}\right)\right. \\
& \left.+\sum_{n=1}^{\infty} \log \left(1+e^{-2 \sqrt{2 n e B} \beta}+2 \cosh (\mu \beta) e^{-\sqrt{2 n e B} \beta}\right)\right\},
\end{aligned}
$$

which is continuous, even when $\mu$ coincides with an energy level.

When this calculation was finished, it was pointed to us that, in reference 13, the partition function for Dirac fields interacting not only with the background magnetic field, but also among themselves through a Nambu-Jona-Lasinio term, had been obtained through a different regularization (see also [14]; for a calculation of paritybreaking corrections, see [15]). However, in the absence of this last interaction, the result in such reference differs from the present one for two reasons: In the first place, the authors of [13] were considering a reducible representation of the gamma matrices, which lead them to an overall factor of 2 with respect to (62). But, more important, in the same reference the determinant of the Dirac operator was evaluated as the square root of minus the squared Dirac operator, thus not including the factor coming from the phase of the determinant or, equivalently, from the multiplicative anomaly [16, which we did consider (see equation (28)). As we will discuss in detail in section 6 , this leads to completely different predictions regarding the Hall conductivity. 


\section{Free energy and particle density}

From equation (62), the free energy per unit area $\left(F=-\frac{1}{\beta} \log Z\right)$ can be obtained $\boldsymbol{~}$. It is given by

$$
\begin{aligned}
F(\mu, B, \beta) & =-\Delta_{L}\left\{\frac{1}{\beta} \log \left(2 \cosh \left(\frac{\mu \beta}{2}\right)\right)-\frac{|\mu|}{2}+\sqrt{2 e B} \zeta_{R}\left(-\frac{1}{2}\right)\right. \\
& \left.+\frac{1}{\beta} \sum_{n=1}^{\infty} \log \left(1+e^{-2 \sqrt{2 n e B} \beta}+2 \cosh (\mu \beta) e^{-\sqrt{2 n e B} \beta}\right)\right\},
\end{aligned}
$$

Moreover, the free energy is continuous at $\mu^{2}=2 k e B, k=0, \ldots, \infty$. In the lowtemperature limit one has

$F\left(2 k e B<\mu^{2}<2(k+1) e B\right) \rightarrow_{\beta \rightarrow \infty}-\Delta_{L}\left\{\sqrt{2 e B}\left(\zeta_{R}\left(-\frac{1}{2}\right)-\sum_{n=1}^{k} \sqrt{n}\right)+k|\mu|\right\}$.

Note that, for $k=0$, this result coincides with the Casimir energy obtained in section 2 even for $\mu \neq 0$, but in this range, i.e., for $\mu$ less than the first Landau level, if positive, or greater than minus the first Landau level, if negative.

The mean particle density can be obtained, also from (62), as $N=\frac{1}{\beta} \frac{d}{d \mu} \log Z$. For nonzero temperature and arbitrary $\mu$ one has

$$
\begin{aligned}
N(\mu, B, \beta) & =\Delta_{L}\left\{\frac{1}{2}\left[\tanh \left(\frac{\mu \beta}{2}\right)-\operatorname{sign}(\mu)\right]\right. \\
& \left.+\sum_{n=1}^{\infty} \frac{2 \sinh (\mu \beta) e^{-\sqrt{2 n e B} \beta}}{1+e^{-2 \sqrt{2 n e B} \beta}+2 \cosh (\mu \beta) e^{-\sqrt{2 n e B} \beta}}\right\},
\end{aligned}
$$

For nonvanishing $\mu$, the low-temperature limit differs, depending on the $\mu$-range considered

$$
N\left(2 e k B<\mu^{2}<2 e(k+1) B\right) \rightarrow_{\beta \rightarrow \infty} k \Delta_{L} \operatorname{sign}(\mu),
$$

where, as before, $k=\left[\frac{\mu^{2}}{2 e B}\right]$.

This result was to be expected for particles with the statistic of fermions, since relativistic field theory naturally leads to the spin-statistics theorem. At zero temperature, $\mu$ is nothing but the Fermi energy; for example, for $\mu>0$, as $\mu$ grows past a Landau level, such level becomes entirely filled.

From the previous result, the mean value of the particle density at zero temperature can be obtained. After recovering units, one has

$$
j^{0}\left(2 e c^{2} \hbar B k<\mu^{2}<2 e B c^{2} \hbar(k+1)\right)=\frac{-k c e^{2} B}{h} \operatorname{sign}(\mu),
$$

the other two components of the current density tri-vector being equal to zero in the absence of an electric field. Now, the zero-temperature limit of the same trivector in the presence of crossed homogeneous electric $\left(F^{\prime}\right)$ and magnetic $\left(B^{\prime}\right)$ fields can retrieved, for $F^{\prime}<c B^{\prime}$, by performing a Lorentz boost with absolute value of the velocity $\frac{F^{\prime}}{B^{\prime}}$. Suppose, for definiteness, that the homogeneous electric field points along the positive $y$ axis. Then, the velocity of the Lorentz boost must point along the negative $x$-axis, and the transformation gives as a result

$$
j^{\prime 0}=\frac{-n c e^{2} B^{\prime}}{h} \operatorname{sign}(\mu), \quad j^{\prime x}=\frac{-n e^{2} F^{\prime}}{h} \operatorname{sign}(\mu), \quad j^{\prime y}=0 .
$$

I Consistently with the comments in previous sections, all the results in this section and in the rest of this paper are independent from the representation of the gamma matrices chosen. 
As a consequence, the quantized zero-temperature Hall conductivity is

$$
\sigma_{x y}=\frac{-n e^{2}}{h} \operatorname{sign}(\mu)
$$

\section{Final comments}

A comment is in order concerning the zero temperature value of the particle density in equation (64) when the chemical potential coincides with an energy level, i.e., for $\mu^{2}=2 e k B, k=0, \ldots, \infty$ In all these cases, the operation of setting $\mu$ to its value doesn't commute with that of taking the $\beta \rightarrow \infty$ limit.

For instance, in the case $\mu=0, N(0, B, \beta)$ is undefined at all temperatures, while $\lim _{\mu \rightarrow 0}\left[\lim _{\beta \rightarrow \infty} N\left(\mu^{2}<2 e B, B, \beta\right)\right]=0$.

On the other hand, each time $\mu^{2}=2 e k B, \quad k=1, \ldots, \infty$, one has $\lim _{\beta \rightarrow \infty} N( \pm \sqrt{2 e k B}, B, \beta)= \pm \Delta_{L}\left(k-\frac{1}{2}\right)$, while $\lim _{\mu \rightarrow \pm \sqrt{2 e k B}}\left[\lim _{\beta \rightarrow \infty} N(\mu, B, \beta)\right]$ is undetermined.

As already commented in the Introduction, after this calculation was finished, reference [4] was brought to our attention. Their prediction concerning the Hall conductivity differ from ours. In fact, the Hall conductivity in that paper contains an overall factor of 4 with respect to ours, which is due to the use of a reducible representation and an extra sum over two values of spin. This is a more or less trivial difference. But, more important, after this factor is removed, their Hall conductivity is quantized in half-integer units of magnetic flux. This originates the word "unconventional" in the title of [4. Our calculation, instead, leads to a Hall conductivity quantized in terms of integer quanta of flux density, which is entirely conventional, but obtained here from very first principles. The difference between both results is due to the inclusion, in our calculation, of the phase of the determinant (or, equivalently in this case, of the multiplicative anomaly). The interesting comment at this point is that experimental results on the Integer Quantum Hall Effect can clarify the physical relevance of the multiplicative anomaly (some recent results [17, 18, seemingly favor "unconventional" quantization).

Finally, we mention that the more realistic case of massive fermions is at present under study [19].

\section{Acknowledgments}

We thank Professors Paola Giacconi, Gerardo Rossini and Roberto Soldati for useful discussions. We also thank Professor V.P. Gusynin for bringing references [4] and [13] to our knowledge.

This work was partially supported by Universidad Nacional de La Plata, under Grant 11/X381.

\section{References}

[1] K. v. Klitzing. The quantized Hall effect. Rev. Mod. Phys., 58:519, 1986.

[2] T. Chakraborty and P. Pietiläinen. Quantum Hall effects: Integral and fractional. SpringerVerlag, Berlin, 1995.

[3] Y. Zheng and T. Ando. Hall conductivity of a two-dimensional graphite system. Phys. Rev B, 65:245420, 2002. 
[4] V.P. Gusynin and S.G. Sharapov. Unconventional Integer Quantum Hall effect in graphene. Phys. Rev. Lett., 95:146802, 2005.

[5] C.G. Beneventano and E.M. Santangelo. Relastivistic Landau problem at finite temperature. Proceedings of the Seventh International Workshop Quantum Field Theory under the Influence of External Conditions, QFEXT'05, Submitted to Jour. Phys. A: Math. Gen., Special Issue, 2005.

[6] L.S. Gradshteyn and L.M. Ryzhik. Table of Integrals, Series and Products. Academic Press, USA, 2000.

[7] E. Elizalde. Ten physical applications of spectral zeta functions. Springer-Verlag, Berlin, 1995.

[8] A.J. Niemi and G.W. Semenoff. Fermion Number Fractionization in Quantum Field Theory. Phys.Reports, 135:99, 1986.

[9] A. Actor. Chemical Potentials in Gauge Theories. Phys.Lett., B157:53, 1985.

[10] J.S. Dowker and R. Critchley. Effective Lagrangian and energy momentum tensor in de Sitter space. Phys. Rev., D13:3224, 1976.

[11] C.G. Beneventano and E.M. Santangelo. Finite temperature properties of the Dirac operator under local boundary conditions. Jour. Phys. A: Math. Gen., 37:9261, 2004.

[12] G. Cognola, E. Elizalde and S. Zerbini. Dirac Functional Determinants in Terms of the Eta Invariant and the Noncommutative Residue. Commun.Math.Phys. 237:507, 2003.

[13] V.P. Gusynin, V.A. Miranski and I.A. Shorkovy. Dynamical flavor symmetry breaking by a magnetic field in 2+1 dimensions. Phys. Rev. D 52:4718, 1995.

[14] A.S. Vshivtsev, B.V. Magnitskii, V.C. Zhukowskii and K.B. Klimenko. Dynamical effects in (2+1)-dimensional theories with four-fermion interaction. Phys. Part. Nucl. 29:523, 1988.

[15] D.C. Cabra and G.L. Rossini. On planar fermions with quartic interaction at finite temperature and density. Eur. Phys. J. B 29:529, 2002.

[16] M. Kontsevich and S. Vishik. Geometry of determinants of elliptic operators. Functional Analysis on the Eve of the 21st Century, Vol. 1, 173. New Brunswick, NJ, 1993.

[17] K.S. Novoselov, A.K. Geim, S.V. Morozov,D. Jiang, M.I. Katsnelson, I.V. Grigorieva, S.V. Dubonos and A.A. Firsov. Two-dimensional gas of massless Dirac fermions in graphene. Nature 438:197, 2005.

[18] Y. Zhang, Y-W. Tang, H.L. Stormer and P. Kim. Experimental observation of the quantum Hall effect and Berry's phase in graphene. Nature 438:201, 2005.

[19] C.G. Beneventano, P. Giacconi, E.M. Santangelo and R. Soldati. Work in progress. 2005. 